Bangladesh J. Zool. 42(2): 141-152, 2014

\title{
AVIFAUNA OF PASHUKHALI AND GAJDHAR VILLAGE IN NETROKONA DISTRICT, BANGLADESH
}

\author{
Shawkat Imam Khan*, Habibon Naher ${ }^{1}$ and Muhammad Saiful Islam ${ }^{1}$ \\ Department of Natural History, Bangladesh National Museum, \\ Shahbag, Dhaka-1000, Bangladesh
}

\begin{abstract}
Avifauna of village Pashukhali and Gajdhar in the district of Netrokona, Bangladesh was carried out during July 2003-June 2004. A total of 101 species of birds belonging to 12 orders, 35 families and 82 genera were recorded. Out of 101 species, $61(60.4 \%)$ were non-passerine and 40 (39.6\%) were passerine. Among them, $80(79.2 \%)$ were resident and $21(20.8 \%)$ were migratory birds. Of the nonpasserine birds, $15(24.6 \%)$ were migratory and 46 were $(75.4 \%)$ resident. While in passerine birds, $6(15 \%)$ were migratory and $34(85 \%)$ were resident. Of the total species, $18(17.8 \%)$ were locally found to be very common, 41 (40.6\%) were common, $29(28.7 \%)$ fairly common and $13(12.9 \%)$ rare or few. Out of 101 species, highest numbers $(58.4 \%)$ of species were widely distributed. Maximum numbers of birds $(78.2 \%)$ were terrestrial.
\end{abstract}

Key words: Avifauna, diversity, Netrokona.

\section{INTRODUCTION}

Birds are an extraordinary creature which covers different types of ecosystems. It plays a significant role in pollination, seed dispersal, scavenging and pest control. There are 632 species of birds of which, 388 are resident and 240 are migratory (IUCN 2000) in Bangladesh. Due to habitat destruction and human disturbance, the population of birds is decreasing at an alarming rate. Every county should have a list of birds, because they are important biological components of all ecosystems (Ahsan and Khanom 2005). As found in every ecosystem, they are highly susceptible to any change in their habitat and hence they are good biological indicators and are used in bioassays (Ahsan and Khanom 2005).

Different workers carried out studies on avifauna of Bangladesh in different parts of the country (Islam 1970, Khan 1982, 2005, Sarker and Sarker 1988, Thompson and Jhonson 2003, Khan and Naher 2009). But a little or no attempt was made to study the diversity, status, habitat and distribution of birds in Netrokona district, an area supposed to be rich in avifauna due to its topography and other ideal geographical and beneficial ecological situation. The main objectives of this work were to make a list of bird species. In addition measuring the abundance of migratory species and their status, local distribution and habitat of birds are presented.

*Author for correspondence: <shawkat194@gmail.com>. 1Department of Zoology, Jagannath University, Dhaka-1100, Bangladesh. 


\section{MATERIAL AND METHODS}

A year long (July 2003 - June 2004) survey was conducted out at the village Pashukhali and Gajdhar under the Upazilla Mohanganj in the district of Netrokona (Fig. 1). The study area is surrounded by Meghalaya of India to the north, native district Sunamganj, Kishorganj and Mymensingh border the east, south and west of Netrokona respectively. The river Kangsa separated the village Pachuakhai from the Upazilla Dharampasha of Sunamganj district to the northern side and the river Dhanu is on the eastern side of the village Gajdhar.

The field survey was conducted fortnightly between $0530 \mathrm{hr}$ to $1800 \mathrm{hr}$. Minimum four days were spent in each visit. The time schedule fluctuated depending on the seasonal variation. All types of habitats (i.e., open field, paddy field, village area, bridle paths, rivers, river sides, canal, pond etc.) were covered under the study. The area was visited on foot. Sometimes non-motorized boat was used to cover the river, canal and wetland areas. The muddy area was surveyed on foot for wader group.

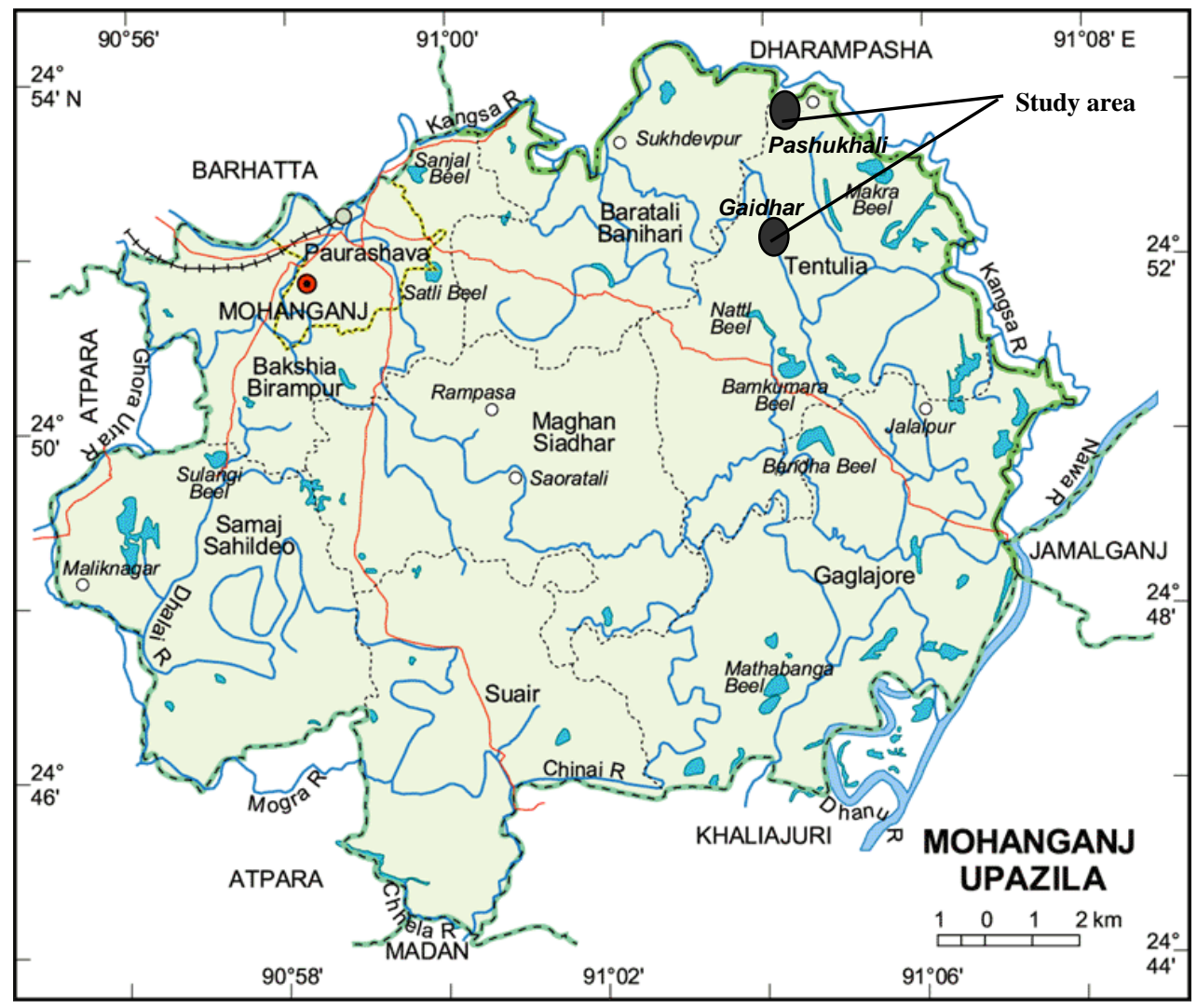

Fig. 1. Map of Mohanganj Upazila showing the locations of the study area. 
Direct observations were made, sometimes binoculars to conduct the study. Bird species were identified by using standard books (Ali and Ripley 1983, 1987, Ali 1996, Grimmett et al. 1999). For the convenience of the habitat study, areas were divided into eight types i.e., Open area (O), Paddy field (Pf), Bush (B), Tree $(\mathrm{T})$, Water-edges $(\mathrm{We})$, Village area $(\mathrm{V})$, River edge $(\mathrm{Re})$ and Pond $(\mathrm{P})$. The local status of the birds, i.e., very common $(\mathrm{Vc})$, common $(\mathrm{C})$, fairly common $(\mathrm{Fc})$ and few or rare (F) were ascertained on the basis of methods used by Khan (1982). The status categories are: Very Common $(\mathrm{Vc})$ - bird species which were recorded on $80-100 \%$ of the field visits; Common (C) - species that were observed on 50$79 \%$ of the field visits; Fairly Common $(\mathrm{Fc})$ - species that were found on $20-49 \%$ of the field visits and Few or Rare $(\mathrm{F})$ - species that were found on less than $19 \%$ of the visits. Tree canopy used by the birds was recorded during observations.

\section{RESULTS AND DISCUSSION}

A total of 101 species (Table 3) of birds were recorded at the village of Pashukhali and Gajdhar in the district of Netrokona. It was about $16 \%$ of the total bird species (i.e., 632) in Bangladesh (IUCN 2000). The observed species belonged to 12 orders, 35 families and 82 genera. The recorded species comprised $61(60.4 \%)$ non-passerine and 40 (39.6\%) passerine birds. Among 101 species, $80(79.2 \%)$ were resident and $21(20.8 \%)$ were migratory, that is, the resident species were nearly four times higher than the migratory ones. Out of 61 non-passerine birds, $15(24.6 \%)$ were migratory and $46(75.4 \%)$ were resident, while among passerines, 6 (15\%) were migratory and $34(85 \%)$ were resident.

The largest number of bird species (40 i.e., 39.6\%) and genera (31 i.e., $37.8 \%)$ belonged to the order Passeriformes and the smallest number of species (1 i.e., 0.9\%) and genera (1 i.e., 1.2\%) to Upupiformes and Psittaciformes respectively. Among the recorded species, family Corvidae (order Passeriformes) comprised the highest number of species (8 i.e., $7.9 \%$ of the total and $20 \%$ of the passerines) and genera (6 i.e., $7.3 \%$ of the total and $19.4 \%$ of the passerines). As many as $13(37.1 \%)$ families (9 i.e., 69.2\% non-passerines and 4 i.e., 30.8\% passerines) were represented by single species each.

Among the non-passerine species, both of the family Accipitridae and Ardeidae contained the highest number of genera (5 i.e., $6.1 \%$ of the total and $9.8 \%$ of the non-passerines), while each of the family Ardeidae, Accipitridae, Charadriifdae, Scolopacidae and Centropodidae had the highest number of species (5 i.e., $5 \%$ of the total and $8.2 \%$ of the non-passerines). The single genus Vanellus (Charadriidae: Ciconiformes) composed of the highest number of species (4 i.e., $3.7 \%$ of the total and $6.6 \%$ of the non-passerines). 
During the study period, $18(17.8 \%)$ species were found to be very common, $41(40.6 \%)$ species common, $29(28.7 \%)$ fairly common and $13(12.9 \%)$ were rare or few. Among very common species $12(66.7 \%)$ species were passerines and 6 $(33.3 \%)$ were non-passerines. All the very common species were resident. Of the recorded common species, $16(39 \%)$ were passerines, $25(61 \%)$ were nonpasserines and 3 \{7.3\% i.e., 1 (33.3\%) passerines and 2 (66.7\%) non-passerines\} were migratory, while 38 \{92.7\% i.e., 15 (39.5\%) passerines and $23(60.5 \%)$ nonpasserines\} were resident birds. On the other hand, among the fairly common species, $8(27.6 \%)$ were passerines and $21(72.4 \%)$ were non-passerines, of which 13 \{44.8\% i.e., $2(15.4 \%)$ passerines and 11 (84.6\%) non-passerines\} were migratory and $16\{55.2 \%$ i.e., $6(37.5 \%)$ passerines and $10(62.5 \%)$ nonpasserines\} were resident. In case of rare or few species, $4(30.8 \%)$ were passerines and $9(69.2 \%)$ were non-passerines, of which 4 \{30.8\% i.e., $3(75 \%)$ passerines and 1 (25\%) non-passerines\} were migratory and $9\{69.2 \%$ i.e., 1 $(11 \%)$ passerines, $8(89 \%)$ non-passerines\} were resident. It was noticed that Pallas's Fish Eagle was common in the study area, which is remarked as critically endangered species in Bangladesh (IUCN 2000).

Table 1. Distribution of birds in Pashukhali and Gajdhar village.

\begin{tabular}{lcccccccc}
\hline Area & $\begin{array}{c}\text { Non- } \\
\text { passerine }\end{array}$ & Passerine & Total & $\begin{array}{c}\text { \% of total } \\
\text { recorded } \\
\text { species }\end{array}$ & M & $\begin{array}{c}\% \text { of total } \\
\text { migratory } \\
\text { species } \\
\text { recorded }\end{array}$ & $\begin{array}{c}\text { R } \\
\text { \% of total } \\
\text { resident } \\
\text { species } \\
\text { recorded }\end{array}$ \\
\hline G & 26 & 10 & 36 & 35.6 & 5 & 23.8 & 31 & 38.8 \\
P & 6 & 0 & 6 & 6 & 3 & 14.3 & 3 & 3.7 \\
G \& P & 29 & 30 & 59 & 58.4 & 13 & 61.9 & 46 & 57.5 \\
\hline
\end{tabular}

Note: G - Gajdhar, P - Pashukhali, M - Migratory, R - Resident.

Table 2. Percentage of bird species in different habitats.

\begin{tabular}{lcccccccc}
\hline Habitat & Total & $\mathrm{P}$ & $\mathrm{NP}$ & $\begin{array}{c}\text { \% of } \\
\text { total }\end{array}$ & $\mathrm{M}$ & $\begin{array}{c}\text { \% of total } \\
\text { migratory species } \\
\text { recorded }\end{array}$ & $\begin{array}{c}\mathrm{R} \\
\text { \% of total } \\
\text { resident species } \\
\text { recorded }\end{array}$ \\
\hline Aquatic & 5 & 0 & 5 & 5 & 3 & 14.2 & 2 & 2.5 \\
Semi-aquatic & 17 & 0 & 17 & 16.8 & 9 & 42.9 & 8 & 10 \\
Terrestrial & 79 & 40 & 39 & 78.2 & 9 & 42.9 & 70 & 87.5 \\
\hline
\end{tabular}

Note: P - Passerine, NP - Non-passerine, M - Migratory, R - Resident

From the study, it was found that the species distribution was not the same throughout the area. Out of 101 species, 59 (58.4\%) were widely distributed throughout the area. From the remaining $42(41.6 \%)$ species, $36(88.7 \%)$ were 
Table 3. Bird species recorded at Pashukhali and Gajdhar in Netrokona district, Bangladesh.

\begin{tabular}{|c|c|c|c|c|c|c|c|c|c|c|}
\hline \multirow[b]{2}{*}{ Scientific Name } & \multirow[b]{2}{*}{ English Name } & \multirow[b]{2}{*}{ Local Name } & \multirow[b]{2}{*}{$\mathrm{R} / \mathrm{M}$} & \multirow[b]{2}{*}{$\begin{array}{l}\text { Present } \\
\text { status }\end{array}$} & \multirow{2}{*}{$\begin{array}{l}\text { Local } \\
\text { distri- } \\
\text { bution }\end{array}$} & \multirow[b]{2}{*}{$\begin{array}{l}\text { Habitat } \\
\text { used }\end{array}$} & \multirow[b]{2}{*}{$\begin{array}{l}\text { Canopy } \\
\text { Used }\end{array}$} & \multicolumn{3}{|c|}{ Country status } \\
\hline & & & & & & & & IUCN & SS & RK \\
\hline \multicolumn{11}{|c|}{ Order: Anseriformes Family: Dendrocygnidae } \\
\hline 1. Dendrocygna bicolor (Vieillot 1816) & $\begin{array}{l}\text { Fulvous Whistling- } \\
\text { duck }\end{array}$ & Baro Shorali & $\mathrm{R}$ & $\mathrm{Fc}$ & G & B & $\mathrm{L}$ & NO & $\mathrm{Fc}$ & Uc \\
\hline $\begin{array}{l}\text { 2. Dendrocygna javanica (Horsfield } \\
\text { 1821) }\end{array}$ & $\begin{array}{l}\text { Lesser Whistling- } \\
\text { duck }\end{array}$ & Sharali & $\mathrm{R}$ & $\mathrm{Fc}$ & G & $\mathrm{B}$ & $\mathrm{L}$ & NO & Vc & Vc \\
\hline \multicolumn{11}{|l|}{ Family: Anatidae } \\
\hline 3. Anas acuta (Linnaeus 1758) & Northern Pintail & Lenja Hans & $\mathrm{M}$ & $\mathrm{Fc}$ & G & $\mathrm{B}$ & $\mathrm{L}$ & - & $\mathrm{C}$ & Vc \\
\hline 4. Tadorna ferruginea (Pallas 1764) & Ruddy Shelduck & Chokhachokhi & $\mathrm{M}$ & $\mathrm{Fc}$ & G & $\mathrm{B}$ & $\mathrm{L}$ & - & $\mathrm{Fc}$ & $\mathrm{C}$ \\
\hline 5. Anas quereuedula (Linnaeus 1758) & Gargeny & Giria Hans & $\mathrm{M}$ & Fc & G & $\mathrm{B}$ & $\mathrm{L}$ & - & $\mathrm{Fc}$ & $\mathrm{C}$ \\
\hline \multicolumn{11}{|l|}{ Order: Piciformes Family: Picidae } \\
\hline 6. Dendrocopos macei (Vieillot 1818) & $\begin{array}{l}\text { Fulvous-breasted } \\
\text { Woodpecker }\end{array}$ & $\begin{array}{l}\text { Pakra } \\
\text { Kaththokra }\end{array}$ & $\mathrm{R}$ & $\mathrm{C}$ & $\mathrm{G}$ & $\mathrm{T}$ & M & NO & $\mathrm{Fc}$ & $\mathrm{C}$ \\
\hline 7. Celeus brachyurus (Vieillot 1818) & $\begin{array}{l}\text { Rufous } \\
\text { Woodpecker }\end{array}$ & $\begin{array}{l}\text { Lalchey } \\
\text { Kaththokra }\end{array}$ & $\mathrm{R}$ & $\mathrm{F}$ & $\mathrm{G}$ & $\mathrm{T}$ & M & NO & $\mathrm{F}$ & Uc \\
\hline $\begin{array}{l}\text { 8. Dinopium benghalense (Linnaeus } \\
\text { 1758) }\end{array}$ & $\begin{array}{l}\text { Black-rumped } \\
\text { Flameback }\end{array}$ & Kaththokra & $\mathrm{R}$ & $\mathrm{C}$ & $\mathrm{P}, \mathrm{G}$ & $\mathrm{T}$ & M & NO & $\mathrm{C}$ & Vc \\
\hline \multicolumn{11}{|c|}{ Order: Upupiformes Family: Upupidae } \\
\hline $\begin{array}{l}\text { 9. Upupa eppos (Linnaeus 1758) } \\
\text { Order: Coraciformes Family: Alced }\end{array}$ & & \multicolumn{8}{|c|}{ Order: Coraciformes Family: Alcedinidae } & $\mathrm{C}$ \\
\hline 10. Alcedo atthis (Linnaeus 1758) & $\begin{array}{l}\text { Common } \\
\text { Kingfisher }\end{array}$ & $\begin{array}{l}\text { Choto } \\
\text { Maachranga }\end{array}$ & $\mathrm{R}$ & $\mathrm{Fc}$ & $\mathrm{P}, \mathrm{G}$ & We & $\mathrm{L}, \mathrm{M}$ & NO & $\mathrm{C}$ & $\mathrm{C}$ \\
\hline 11. Alcedo meninting (Horsfield 1821) & $\begin{array}{l}\text { Blue-eared } \\
\text { Kingfisher }\end{array}$ & $\begin{array}{l}\text { Nilkan } \\
\text { Maachranga }\end{array}$ & $\mathrm{R}$ & $\mathrm{Fc}$ & $\mathrm{G}$ & We & $\mathrm{L}, \mathrm{M}$ & NO & $\mathrm{F}$ & - \\
\hline $\begin{array}{l}\text { 12. Halcyon smyrnensis (Linnaeus } \\
1758 \text { ) }\end{array}$ & $\begin{array}{l}\text { White-throated } \\
\text { Kingfisher }\end{array}$ & $\begin{array}{l}\text { Sada-book } \\
\text { Maachranga }\end{array}$ & $\mathrm{R}$ & $\mathrm{C}$ & $\mathrm{G}$ & $\mathrm{T}, \mathrm{We}$ & $\mathrm{M}, \mathrm{U}$ & NO & $\mathrm{C}$ & Vc \\
\hline \multicolumn{11}{|l|}{ Family: Meropidae } \\
\hline 13. Merops orientalis (Latham 1801) & Green Bea-eater & Suichora & $\mathrm{R}$ & $\mathrm{C}$ & G & $\mathrm{T}$ & $\mathrm{M}, \mathrm{U}$ & NO & $\mathrm{C}$ & Vc \\
\hline $\begin{array}{l}\text { Order: Cuculiformes Family: Centrop } \\
\text { 14. Centropus bengalensis (Gmelin } \\
\text { 1788) }\end{array}$ & $\begin{array}{l}\text { didae } \\
\text { Lesser Coucal }\end{array}$ & $\begin{array}{l}\text { Choto } \\
\text { Kanakuka }\end{array}$ & $\mathrm{R}$ & $\mathrm{C}$ & $\mathrm{G}$ & $\mathrm{V}, \mathrm{Bu}$ & $\mathrm{L}, \mathrm{M}$ & NO & $\mathrm{Fc}$ & $\mathrm{C}$ \\
\hline $\begin{array}{l}\text { 15. Centropus sinensis (Stephens } \\
\text { 1815) }\end{array}$ & Greater Coucal & Baro Kanakuka & $\mathrm{R}$ & $\mathrm{C}$ & G & $\mathrm{V}, \mathrm{Bu}$ & $\mathrm{L}, \mathrm{M}$ & NO & Vc & $\mathrm{C}$ \\
\hline 16. Cuculus micropterus (Gould 1837) & Indian Cuckoo & Bou-katha-kao & $\mathrm{R}$ & $\mathrm{C}$ & $\mathrm{G}$ & $\mathrm{T}$ & $\mathrm{M}, \mathrm{U}$ & NO & $\mathrm{Fc}$ & $\mathrm{C}$ \\
\hline $\begin{array}{l}\text { 17. Eudynamys scolopacea (Linnaeus } \\
1758 \text { ) }\end{array}$ & Asian Cuckoo & Kokil & $\mathrm{R}$ & $\mathrm{Fc}$ & G & $\mathrm{T}$ & $\mathrm{M}, \mathrm{U}$ & NO & $\mathrm{Fc}$ & Vc \\
\hline 18. Hierococcyx varius (Vahl 1797) & $\begin{array}{l}\text { Common Hawk } \\
\text { Cuckoo }\end{array}$ & Chokhgelo & $\mathrm{R}$ & $\mathrm{C}$ & $\mathrm{P}, \mathrm{G}$ & $\mathrm{T}$ & $\mathrm{M}, \mathrm{U}$ & NO & $\mathrm{C}$ & Vc \\
\hline
\end{tabular}


Table 3 contd.

Order: Psittaciformes Family: Psittacidae

19. Psittacula krameri (Scopoli 1769) Rose-ringed

Parakeet

Order: Apodiformes Family: Apodidae

20. Apus naffinis (J.E. Gray 1830)

21. Cypsiurus balasiensis (J.E. Gray 1829)

Family: Hemiprocnidae

22. Hemiprocne coronata (Tickell Crested Treeswift 1833 )

Order: Strigiformes Family: Tytonidae

23. Phodilus badius (Horsfield 1821) Oriental Bay Owl

24. Tyto alba (Scopoli 1769)

Barn Owl

Family: Strigidae

25. Athene brama (Temminck 1821)

26. Ninox scutulata (Raffles 1822)

27. Ketupa zeylonensis (Gmelin 1788)

28. Otus scops (Linnaeus 1758)

Order: Columbiformes Family: Columbida

29. Columba livia (Gmelin 1789)

30. Streptopelia chinensis (Scopoli 1786)

31. Streptopelia decaocto (Frivaldszky 1838)

32. Streptopelia orientalis (Latham 1790)

Order: Gruifoemes Family: Rallidae

33. Amaurornis phoenicurus (Pennant 1769)

34. Gallicrex cinerea (Gmelin 1789)

35. Gallinula chloropus (Linnaeus 1758)

36. Porphyrio porphyrio (Linnaeus 1758)

\section{Spotted Owlet}

Brown Hawk Owl

Brown Fish Owl

Eurasian Scops Owl

Rock Pigeon

Spotted Dove

Eurasian Collered

Dove

Oriental Turtle

Dove

White-breasted

Waterhen

Watercock

Common

Moorhen

Purple

Swamphen
Tia

(1)

Ababil

Nakkati

Jutial

Gasbatasi

Pencha

Laxmi Pencha

Khuruley

Pencha

Kupokh

Bhutum

Pencha

Nimpokh

Jalali Kobutar

Tila Ghughu

Raj Ghughu

Ghughu

Dahuk

Kura

Jol Moorgi

Kalim

\section{G}

C

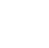

$\begin{array}{ll}\mathrm{R} & \mathrm{C} \\ \mathrm{R} & \mathrm{VC}\end{array}$

\section{P,G}

$P, G$

$$
\mathrm{R}
$$

F

$\mathrm{R}$

R C

R F

$\begin{array}{ll}R & F \\ R & F\end{array}$

$\mathrm{R}$

F

$\mathrm{R} \quad \mathrm{Vc}$

$\begin{array}{ll}\mathrm{R} & \mathrm{Vc} \\ \mathrm{R} & \mathrm{Vc}\end{array}$

$\mathrm{R} \quad \mathrm{Fc}$

M Fc

R C

$\mathrm{R} \quad \mathrm{FC}$

$\mathrm{R} \quad \mathrm{FC}$

$\mathrm{R}$

C

\section{$\mathrm{P}, \mathrm{G}$}

G

$\mathrm{P}, \mathrm{G}$

P,G

$\mathrm{P}$

Pf

P,Pf

Pf

O,Pf
T,O O,Pf
Pf
P,Pf
Pf

U

$\begin{array}{llll}\mathrm{U} & \mathrm{NO} & \mathrm{Vc} & \mathrm{Vc} \\ \mathrm{U} & \mathrm{NO} & \mathrm{Fc} & \mathrm{Vc}\end{array}$

U

U

$$
\text { U }
$$

U

U

U

U

L,U

L,U

L,U

L,U

L

L
L

L

$\begin{array}{lll}\text { NO } & \text { C } & \text { Vc } \\ \text { NO } & \text { C } & \text { C } \\ \text { NO } & \text { Fc } & \text { C } \\ \text { NO } & \text { C } & \text { C }\end{array}$


Table 3 contd.

\begin{tabular}{|c|c|c|c|c|c|c|c|c|c|c|}
\hline \multicolumn{11}{|c|}{ Order: Ciconiiformes Family: Scolopacidae } \\
\hline $\begin{array}{l}\text { 37. Actitis hypoleucos (Linnaeus } \\
\text { 1758) }\end{array}$ & $\begin{array}{l}\text { Common } \\
\text { Sandpiper }\end{array}$ & Pati Batan & M & Fc & $\mathrm{P}, \mathrm{G}$ & $\mathrm{Re}, \mathrm{We}$ & $\mathrm{L}$ & - & $\mathrm{F}$ & $\mathrm{C}$ \\
\hline $\begin{array}{l}\text { 38. Gallinago gallinago (Linnaeus } \\
\text { 1758) }\end{array}$ & Fantail Snipe & Kada-khocha & M & $\mathrm{Fc}$ & $\mathrm{P}, \mathrm{G}$ & $\mathrm{O}, \mathrm{We}$ & $\mathrm{L}$ & - & $\mathrm{Fc}$ & $\mathrm{C}$ \\
\hline $\begin{array}{l}\text { 39. Gallinago stenura (Bonaparte } \\
\text { 1830) }\end{array}$ & Pintail Snipe & Kada-khocha & M & $\mathrm{C}$ & $\mathrm{P}, \mathrm{G}$ & $\mathrm{O}, \mathrm{We}$ & $\mathrm{L}$ & - & $\mathrm{Fc}$ & $\mathrm{C}$ \\
\hline 40. Limosa limosa (Linnaeus 1758 ) & $\begin{array}{l}\text { Black-tailed } \\
\text { Godwit }\end{array}$ & Jurali & M & $\mathrm{Fc}$ & $\mathrm{P}, \mathrm{G}$ & $\mathrm{O}$ & $\mathrm{L}$ & - & $\mathrm{F}$ & $\mathrm{C}$ \\
\hline 41. Tringa totanus (Linnaeus 1758 ) & $\begin{array}{l}\text { Common } \\
\text { Redshank }\end{array}$ & Lal-pa Pi-oo & $\mathrm{M}$ & $\mathrm{F}$ & $\mathrm{P}$ & $\mathrm{O}, \mathrm{We}$ & $\mathrm{L}$ & - & $\mathrm{F}$ & $\mathrm{C}$ \\
\hline \multicolumn{11}{|l|}{ Family: Charadriidae } \\
\hline $\begin{array}{l}\text { 42. Charadrius hiaticula (Linnaeus } \\
1758 \text { ) }\end{array}$ & $\begin{array}{l}\text { Common Ringed } \\
\text { Plover }\end{array}$ & Pati Nathjiria & $\mathrm{M}$ & $\mathrm{Fc}$ & $\mathrm{P}$ & $\mathrm{O}$ & $\mathrm{L}$ & - & $\mathrm{Fc}$ & $\mathrm{C}$ \\
\hline 43. Vanellus cinereus (Blyth 1842) & $\begin{array}{l}\text { Grey-headed } \\
\text { Lapwing }\end{array}$ & Dhushor ti-ti & M & $\mathrm{Fc}$ & $\mathrm{P}, \mathrm{G}$ & $\mathrm{O}$ & $\mathrm{L}$ & - & $\mathrm{C}$ & $\mathrm{C}$ \\
\hline $\begin{array}{l}\text { 44. Vanellus vanellus (Linnaeus } \\
\text { 1758) }\end{array}$ & Northern Lapwing & Uttare ti-ti & M & $\mathrm{F}$ & $\mathrm{P}, \mathrm{G}$ & $\mathrm{O}$ & $\mathrm{L}$ & - & $\mathrm{O}$ & $\mathrm{r}$ \\
\hline 45. Vanellus indicus (Boddaert 1783) & $\begin{array}{l}\text { Red-wattled } \\
\text { Lapwing }\end{array}$ & $\begin{array}{l}\text { Lal-lotika Hot- } \\
\text { ti-ti }\end{array}$ & $\mathrm{R}$ & $\mathrm{C}$ & $\mathrm{P}$ & $\mathrm{O}$ & $\mathrm{L}$ & NO & Vc & $\mathrm{C}$ \\
\hline $\begin{array}{l}\text { 46. Vanellus malabaricus (Boddaert } \\
\text { 1783) }\end{array}$ & $\begin{array}{l}\text { Yellow-wattled } \\
\text { Lapwing }\end{array}$ & Hot-ti-ti & $\mathrm{R}$ & $\mathrm{C}$ & $\mathrm{P}$ & $\mathrm{O}$ & $\mathrm{L}$ & NO & $\mathrm{Fc}$ & $\mathrm{C}$ \\
\hline \multicolumn{11}{|l|}{ Family: Glareolidae } \\
\hline 47. Pluvialis dominicus (Gmelin 1789) & $\begin{array}{l}\text { Pacific Golden } \\
\text { Plover }\end{array}$ & Batan & M & $\mathrm{C}$ & $\mathrm{P}$ & $\mathrm{We}, \mathrm{O}$ & $\mathrm{L}$ & - & $\mathrm{Fc}$ & $\mathrm{Vc}$ \\
\hline $\begin{array}{l}\text { Family: Rostratulidae } \\
\text { 48. Rostratula bengalensis (Linnaeus } \\
\text { 1758) }\end{array}$ & $\begin{array}{l}\text { Greater Painted- } \\
\text { snipe }\end{array}$ & Rongila Chaga & $\mathrm{R}$ & $\mathrm{C}$ & $\mathrm{P}$ & $\mathrm{O}$ & $\mathrm{L}$ & NO & $\mathrm{Fc}$ & $\mathrm{C}$ \\
\hline $\begin{array}{l}\text { Family: Laridae } \\
\text { 49. Sterna aurantia (J.E. Gray 1831) }\end{array}$ & River Tern & $\begin{array}{l}\text { Maach } \\
\text { Khaikka }\end{array}$ & $\mathrm{R}$ & $\mathrm{C}$ & $\mathrm{P}, \mathrm{G}$ & $\mathrm{O}$ & $\mathrm{L}$ & NO & $\mathrm{Fc}$ & $\mathrm{Vc}$ \\
\hline $\begin{array}{l}\text { Family: Accipitridae } \\
\text { 50. Gyps bengalensis (Gmelin 1788) }\end{array}$ & $\begin{array}{l}\text { White-rumped } \\
\text { Vulture }\end{array}$ & Shokun & $\mathrm{R}$ & $\mathrm{F}$ & G & $\mathrm{O}$ & $\mathrm{L}, \mathrm{U}$ & NO & $\mathrm{Fc}$ & Uc \\
\hline $\begin{array}{l}\text { 51. Haliaeetus leucoryphus (Pallas } \\
\text { 1771) }\end{array}$ & $\begin{array}{l}\text { Pallas's Fish } \\
\text { Eagle }\end{array}$ & Kura & $\mathrm{R}$ & $\mathrm{C}$ & G & $\mathrm{T}, \mathrm{O}$ & $\mathrm{L}, \mathrm{U}$ & CR & $\mathrm{O}$ & $\mathrm{r}$ \\
\hline 52. Haliastur indus (Boddaert 1783) & Brahminy Kite & $\begin{array}{l}\text { Shankho } \\
\text { Cheel }\end{array}$ & $\mathrm{R}$ & $\mathrm{Vc}$ & P.G & $\mathrm{T}, \mathrm{O}$ & $\mathrm{L}, \mathrm{U}$ & NO & $\mathrm{C}$ & $\mathrm{C}$ \\
\hline 53. Milvus migrans (Boddaert 1783) & Black Kite & Bhubon Cheel & $\mathrm{R}$ & $\mathrm{C}$ & P.G & $\mathrm{T}$ & $\mathrm{L}, \mathrm{U}$ & NO & Vc & Uc \\
\hline $\begin{array}{l}\text { 54. Circus melanoleucos (Pennant } \\
\text { 1769) }\end{array}$ & Pied Harrier & Pakra Patabaz & $\mathrm{M}$ & $\mathrm{Fc}$ & $\mathrm{P}, \mathrm{G}$ & $\mathrm{T}$ & $\mathrm{L}, \mathrm{U}$ & - & $\mathrm{O}$ & $\mathrm{C}$ \\
\hline
\end{tabular}


Table 3 contd.

\begin{tabular}{|c|c|c|c|c|c|c|c|c|c|c|}
\hline \multicolumn{11}{|l|}{ Family: Falconidae } \\
\hline $\begin{array}{l}\text { 55. Falco tinnunculus (Linnaeus } \\
\text { 1758) }\end{array}$ & Common Kestrel & Pati Kestrel & M & $\mathrm{Fc}$ & $\mathrm{P}, \mathrm{G}$ & $\mathrm{O}$ & $\mathrm{L}, \mathrm{U}$ & - & $\mathrm{F}$ & $\mathrm{C}$ \\
\hline \multicolumn{11}{|l|}{ Family: Phalacrocoracidae } \\
\hline 56. Phalacrocorax niger (Vieillot 1817) & Little cormorant & $\begin{array}{l}\text { Choto Paan- } \\
\text { kowri }\end{array}$ & $\mathrm{R}$ & $\mathrm{C}$ & $\mathrm{G}$ & $\mathrm{P}, \mathrm{T}$ & $\mathrm{L}, \mathrm{M}$ & NO & $\mathrm{C}$ & Vc \\
\hline \multicolumn{11}{|l|}{ Family: Ardeidae } \\
\hline 57. Ardeola grayii (Sykes 1832) & $\begin{array}{l}\text { Indian pond } \\
\text { Heron }\end{array}$ & Kani Bok & $\mathrm{R}$ & $\mathrm{Vc}$ & $\mathrm{P}, \mathrm{G}$ & $\begin{array}{l}\text { Re,P,O, } \\
\text { We }\end{array}$ & $\mathrm{L}$ & NO & $\mathrm{C}$ & Vc \\
\hline 58. Bubulcus ibis (Linnaeus 1758) & Cattle Egret & Go-bok & $\mathrm{R}$ & $\mathrm{C}$ & $P, G$ & $\mathrm{O}, \mathrm{We}$ & $\mathrm{L}$ & NO & Fc & $\mathrm{C}$ \\
\hline 59. Egretta garzetta (Linnaues 1758) & Little Egret & Choto Bok & $\mathrm{R}$ & $\mathrm{Vc}$ & $P, G$ & $\mathrm{P}, \mathrm{We}, \mathrm{O}$ & $\mathrm{L}$ & NO & $\mathrm{C}$ & $\mathrm{C}$ \\
\hline $\begin{array}{l}\text { 60. Lxobrychus cinnamomeus (Gmelin } \\
\text { 1789) }\end{array}$ & Cinnamon Bittern & Lal Bok & $\mathrm{R}$ & $\mathrm{Fc}$ & $\mathrm{P}, \mathrm{G}$ & $\mathrm{Re}, \mathrm{We}, \mathrm{O}$ & $\mathrm{L}$ & NO & $\mathrm{F}$ & $\mathrm{C}$ \\
\hline $\begin{array}{l}\text { 61. Mesophoyx intermedia (Wagler } \\
\text { 1829) }\end{array}$ & $\begin{array}{l}\text { Intermediate } \\
\text { Egret }\end{array}$ & Maijhla Bok & $\mathrm{R}$ & $\mathrm{C}$ & $\mathrm{P}, \mathrm{G}$ & $\mathrm{O}, \mathrm{We}$ & $\mathrm{L}$ & NO & $\mathrm{Fc}$ & Uc \\
\hline $\begin{array}{l}\text { Order: Passeriformes Family: } \\
\text { Laniidae }\end{array}$ & & & & & & & & & & \\
\hline 62. Lanius cristatus (Linnaeus 1758) & Brown Shrike & $\begin{array}{l}\text { Badami } \\
\text { Koshai }\end{array}$ & M & $\mathrm{Fc}$ & $\mathrm{P}, \mathrm{G}$ & $\mathrm{T}, \mathrm{O}$ & $\mathrm{L}, \mathrm{M}$ & - & $\mathrm{C}$ & Vc \\
\hline \multicolumn{11}{|l|}{ Family: Corvidae } \\
\hline $\begin{array}{l}\text { 63. Corvus macrorhynchos (Wagler } \\
\text { 1827) }\end{array}$ & Large-billed Crow & Dar Kak & $\mathrm{R}$ & $\mathrm{C}$ & $\mathrm{P}, \mathrm{G}$ & $\mathrm{T}, \mathrm{V}$ & $\mathrm{L}, \mathrm{M}, \mathrm{U}$ & NO & $\mathrm{C}$ & Vc \\
\hline 64. Corvus splendens (Vieillot 1817) & House Crow & Pati Kak & $\mathrm{R}$ & $\mathrm{Fc}$ & $\mathrm{P}, \mathrm{G}$ & $\mathrm{T}, \mathrm{V}$ & $\mathrm{L}, \mathrm{M}, \mathrm{U}$ & NO & $\mathrm{Vc}$ & Vc \\
\hline $\begin{array}{l}\text { 65. Dendrocitta vagabunda (Latham } \\
1790)\end{array}$ & Rufous Treepie & Harichacha & $\mathrm{R}$ & $\mathrm{C}$ & $\mathrm{P}, \mathrm{G}$ & $\mathrm{T}, \mathrm{V}$ & $\mathrm{M}, \mathrm{U}$ & NO & $\mathrm{Fc}$ & Vc \\
\hline 66. Aegithina tiphia (Linnaeus 1758 ) & Common Iora & Towfik & $\mathrm{R}$ & $\mathrm{C}$ & $P, G$ & $\mathrm{~T}, \mathrm{~V}, \mathrm{Bu}$ & $\mathrm{M}, \mathrm{U}$ & NO & $\mathrm{Vc}$ & Vc \\
\hline $\begin{array}{l}\text { 67. Dicrurus macrocercus (Vieillot } \\
\text { 1817) }\end{array}$ & Black Drongo & Fingey & $\mathrm{R}$ & Vc & $\mathrm{P}, \mathrm{G}$ & $\mathrm{T}, \mathrm{O}$ & $\mathrm{L}, \mathrm{M}$ & NO & $\mathrm{Vc}$ & Vc \\
\hline 68. Dicrurus aeneus (Vieillot 1817) & Bronzed Drongo & Choto Fingey & $\mathrm{R}$ & $\mathrm{C}$ & $\mathrm{P}, \mathrm{G}$ & $\mathrm{T}, \mathrm{O}$ & $\mathrm{L}, \mathrm{M}$ & NO & $\mathrm{Fc}$ & $\mathrm{C}$ \\
\hline 69. Rhipidura albicollis (Vieillot 1818) & $\begin{array}{l}\text { White-throated } \\
\text { Fantail }\end{array}$ & Lejnachani & $\mathrm{R}$ & Vc & $\mathrm{P}, \mathrm{G}$ & $\mathrm{T}, \mathrm{V}$ & $\mathrm{M}$ & NO & $\mathrm{Fc}$ & $\mathrm{C}$ \\
\hline $\begin{array}{l}\text { 70. Oriolus xanthornus (Linnaeus } \\
\text { 1758) }\end{array}$ & $\begin{array}{l}\text { Black-hooded } \\
\text { Oriole }\end{array}$ & Holdey Pakhi & $\mathrm{R}$ & Vc & $\mathrm{P}, \mathrm{G}$ & $\mathrm{T}, \mathrm{V}$ & $\mathrm{M}, \mathrm{U}$ & NO & $\mathrm{C}$ & Vc \\
\hline \multicolumn{11}{|l|}{ Family: Muscicapidae } \\
\hline $\begin{array}{l}\text { 71. Copsychus malabaricus (Scopoli } \\
\text { 1786) }\end{array}$ & $\begin{array}{l}\text { White-rumped } \\
\text { Shama }\end{array}$ & Shama & $\mathrm{R}$ & $\mathrm{C}$ & $\mathrm{G}$ & $\mathrm{T}, \mathrm{Bu}$ & M & NO & $\mathrm{F}$ & $\mathrm{C}$ \\
\hline $\begin{array}{l}\text { 72. Copsychus saularis (Linnaeus } \\
\text { 1758) }\end{array}$ & $\begin{array}{l}\text { Oriental Magpie } \\
\text { Robin }\end{array}$ & Doel & $\mathrm{R}$ & $\mathrm{Vc}$ & $\mathrm{P}, \mathrm{G}$ & $\mathrm{O}, \mathrm{T}$ & $\mathrm{L}, \mathrm{M}$ & NO & $\mathrm{Vc}$ & Vc \\
\hline 73. Luscinia callipoe (Pallas 1776) & $\begin{array}{l}\text { Siberian Ruby } \\
\text { Throat }\end{array}$ & $\begin{array}{l}\text { Siberian } \\
\text { Chunikonthi }\end{array}$ & M & $\mathrm{F}$ & $\mathrm{P}, \mathrm{G}$ & $\mathrm{O}, \mathrm{Pf}$ & $\mathrm{L}, \mathrm{M}$ & - & - & $\mathrm{C}$ \\
\hline
\end{tabular}


Table 3 contd.

Family: Sturnidae

74. Acridotheres fuscus (Wagler 1827)

75. Acridotheres tristis (Linnaeus 1766)

76. Sturnus contra (Linnaeus 1758)

77. Sturnus malabaricus (Gmelin 1789)

Family: Paridae

78. Parus major (Linnaeus 1758)

Family: Pycnonotidae

79. Pycnonotus cafer (Linnaeus 1766)

Family: Cisticolidae

80. Cisticola juncidis (Rafnesque 1810)

81. Prinia gracilis (Lichtenstein 1823)

82. Prinia inornata (Gmelin 1789)

Family: Zosteropidae

83. Zosterops palpebrosus

(Temminck 1824)

Family: Sylviidae

84. Megaurus palustris (Horsfield 1821)

85. Orthotomus sutorius (Pennant 1769)

Jungle Myna

Common Myna

Asian Pied

Starling

Chestnut-tailed

Starling

Great Tit

Red-vented

Bulbul

Zitting Cisticola

Graceful Prinia

Plain Prinia

Oriental White-

eye

Striated

Grassbird

Common

Tailorbird

Jungle Babbler 1823)

Family: Alaudidae

87. Alauda gulgula (Franklin 1831)

88. Ammomanes phoenicurus (Franklin 1831)

89. Eremopterix grisea (Scopoli 1786)

90. Mirafra assamica (Horsfield 1840)

\begin{tabular}{|c|c|c|c|c|c|c|c|c|c|}
\hline Jungle Myna & Jhuti Shalik & $\mathrm{R}$ & $\mathrm{Vc}$ & $\mathrm{P}, \mathrm{G}$ & $\mathrm{T}, \mathrm{O}, \mathrm{V}$ & $\mathrm{L}, \mathrm{M}$ & NO & $\mathrm{C}$ & Vc \\
\hline Common Myna & Bhat Shalik & $\mathrm{R}$ & Vc & $\mathrm{P}, \mathrm{G}$ & $\mathrm{T}, \mathrm{V}$ & $\mathrm{L}, \mathrm{M}$ & NO & Vc & Vc \\
\hline $\begin{array}{l}\text { Asian Pied } \\
\text { Starling }\end{array}$ & Gobrey Shalik & $\mathrm{R}$ & Vc & $\mathrm{P}, \mathrm{G}$ & $\mathrm{T}, \mathrm{V}, \mathrm{O}$ & $\mathrm{L}, \mathrm{M}$ & NO & $\mathrm{C}$ & Vc \\
\hline $\begin{array}{l}\text { Chestnut-tailed } \\
\text { Starling }\end{array}$ & Kath Shalik & $\mathrm{R}$ & Vc & $\mathrm{G}$ & $\mathrm{T}, \mathrm{V}$ & $\mathrm{M}, \mathrm{U}$ & NO & $\mathrm{C}$ & Uc \\
\hline Great Tit & Tit Pockh & $\mathrm{R}$ & $\mathrm{C}$ & $\mathrm{G}$ & $\mathrm{T}, \mathrm{V}, \mathrm{Bu}$ & $\mathrm{M}, \mathrm{U}$ & NO & $\mathrm{C}$ & Vc \\
\hline $\begin{array}{l}\text { Red-vented } \\
\text { Bulbul }\end{array}$ & Bulbuli & $\mathrm{R}$ & $\mathrm{Vc}$ & P.G & $\mathrm{T}, \mathrm{V}$ & $\mathrm{L}, \mathrm{M}, \mathrm{U}$ & NO & Vc & Vc \\
\hline Zitting Cisticola & $\begin{array}{l}\text { Surela } \\
\text { Cisticola }\end{array}$ & $\mathrm{R}$ & $\mathrm{C}$ & $\mathrm{P}, \mathrm{G}$ & $\mathrm{O}$ & $\mathrm{U}$ & NO & $\mathrm{F}$ & $\mathrm{C}$ \\
\hline Graceful Prinia & Ruposhi Prinia & $\mathrm{R}$ & $\mathrm{Fc}$ & $\mathrm{P}, \mathrm{G}$ & $\mathrm{Bu}, \mathrm{O}$ & $\mathrm{L}, \mathrm{M}$ & DD & $\mathrm{Fc}$ & $\mathrm{r}$ \\
\hline Plain Prinia & Pati Prinia & $\mathrm{R}$ & $\mathrm{Fc}$ & $\mathrm{P}, \mathrm{G}$ & $\mathrm{O}, \mathrm{Pf}$ & $\mathrm{M}, \mathrm{U}$ & NO & $\mathrm{F}$ & - \\
\hline $\begin{array}{l}\text { Oriental White- } \\
\text { eye }\end{array}$ & Shet-ankhi & $\mathrm{R}$ & $\mathrm{C}$ & G & $\mathrm{T}, \mathrm{V}$ & $\mathrm{M}, \mathrm{U}$ & NO & $\mathrm{C}$ & $\mathrm{C}$ \\
\hline $\begin{array}{l}\text { Striated } \\
\text { Grassbird }\end{array}$ & Jolar Warbler & $\mathrm{R}$ & $\mathrm{C}$ & $\mathrm{P}, \mathrm{G}$ & $\mathrm{O}, \mathrm{Pf}$ & M & NO & $\mathrm{U}$ & $\mathrm{C}$ \\
\hline $\begin{array}{l}\text { Common } \\
\text { Tailorbird }\end{array}$ & Tuntuni & $\mathrm{R}$ & C & $\mathrm{P}, \mathrm{G}$ & $\mathrm{V}, \mathrm{Bu}$ & $\mathrm{L}, \mathrm{M}$ & NO & $\mathrm{C}$ & Vc \\
\hline Jungle Babbler & Satbhai & $\mathrm{R}$ & $\mathrm{C}$ & G & $\mathrm{V}, \mathrm{Bu}$ & M & NO & $\mathrm{C}$ & Vc \\
\hline Oriental Skylark & Bharat Pakhi & $\mathrm{R}$ & $\mathrm{Fc}$ & $\mathrm{P}, \mathrm{G}$ & $\mathrm{O}$ & M & NO & $\mathrm{F}$ & Uc \\
\hline $\begin{array}{l}\text { Rufous-tailed } \\
\text { Lark }\end{array}$ & Lal Bharat & M & $\mathrm{F}$ & G & $\mathrm{O}$ & M & - & - & - \\
\hline $\begin{array}{l}\text { Ashy-crowned } \\
\text { Sparrow Lark }\end{array}$ & Balu Chata & $\mathrm{R}$ & $\mathrm{F}$ & G & $\mathrm{O}$ & M & NO & $\mathrm{C}$ & $\mathrm{C}$ \\
\hline $\begin{array}{l}\text { Rufous-winged } \\
\text { Bushlark }\end{array}$ & Bharat Pakhi & $\mathrm{R}$ & $\mathrm{Fc}$ & G & $\mathrm{Bu}, \mathrm{O}$ & M & NO & $\mathrm{Fc}$ & Vc \\
\hline
\end{tabular}


Table 3 contd.

\begin{tabular}{|c|c|c|c|c|c|c|c|c|c|c|}
\hline \multicolumn{11}{|l|}{ Family: Nectarinidae } \\
\hline $\begin{array}{l}\text { 91. Arachnothera longirostra } \\
\text { (Latham 1790) }\end{array}$ & $\begin{array}{l}\text { Little } \\
\text { Spiderhunter }\end{array}$ & $\begin{array}{l}\text { Choto } \\
\text { Machorbhuk }\end{array}$ & $\mathrm{R}$ & $\mathrm{Fc}$ & $\mathrm{G}$ & $\mathrm{Bu}, \mathrm{V}$ & $\mathrm{L}, \mathrm{M}$ & NO & $\mathrm{F}$ & $\mathrm{C}$ \\
\hline $\begin{array}{l}\text { 92. Nectarinia asiatica (Latham } \\
\text { 1790) }\end{array}$ & Purple Sunbird & Niltuni & $\mathrm{R}$ & $\mathrm{C}$ & $\mathrm{P}, \mathrm{G}$ & $\mathrm{T}, \mathrm{V}$ & $\mathrm{M}, \mathrm{U}$ & NO & $\mathrm{Vc}$ & Vc \\
\hline $\begin{array}{l}\text { 93. Nectarinia zeylonica (Linnaeus } \\
\text { 1766) }\end{array}$ & $\begin{array}{l}\text { Purple-rumped } \\
\text { Sunbird }\end{array}$ & Moutushi & $\mathrm{R}$ & $\mathrm{C}$ & $\mathrm{P}, \mathrm{G}$ & $\mathrm{T}, \mathrm{V}$ & $\mathrm{M}, \mathrm{U}$ & NO & $\mathrm{Fc}$ & $\mathrm{Vc}$ \\
\hline $\begin{array}{l}\text { 94. Dicaeum erythrorynchos } \\
\text { (Latham 1790) }\end{array}$ & $\begin{array}{l}\text { Pale-billed } \\
\text { Flowerpecker }\end{array}$ & Fuljhuri & $\mathrm{R}$ & $\mathrm{C}$ & G & $\mathrm{T}, \mathrm{V}$ & $\mathrm{M}, \mathrm{U}$ & NO & $\mathrm{Vc}$ & Vc \\
\hline \multicolumn{11}{|l|}{ Family: Passeridae } \\
\hline $\begin{array}{l}\text { 95. Lonchura malacca (Linnaeus } \\
\text { 1766) }\end{array}$ & $\begin{array}{l}\text { Black-headed } \\
\text { Munia }\end{array}$ & $\begin{array}{l}\text { Kalomatha } \\
\text { Munia }\end{array}$ & $\mathrm{R}$ & $\mathrm{C}$ & $\mathrm{P}, \mathrm{G}$ & $\mathrm{T}, \mathrm{O}$ & $\mathrm{L}, \mathrm{M}$ & NO & $\mathrm{F}$ & $\mathrm{Vc}$ \\
\hline $\begin{array}{l}\text { 96. Lonchura punctulata (Linnaeus } \\
\text { 1758) }\end{array}$ & $\begin{array}{l}\text { Scaly-breasted } \\
\text { Munia }\end{array}$ & Tila Munia & $\mathrm{R}$ & Vc & $\mathrm{P}, \mathrm{G}$ & $\mathrm{T}, \mathrm{O}$ & $\mathrm{L}, \mathrm{M}$ & $\mathrm{NO}$ & $\mathrm{C}$ & $\mathrm{Vc}$ \\
\hline $\begin{array}{l}\text { 97. Passer domesticus (Linnaeus } \\
1758 \text { ) }\end{array}$ & House Sparrow & Charui & $\mathrm{R}$ & Vc & $\mathrm{P}, \mathrm{G}$ & $\mathrm{V}, \mathrm{O}$ & $\mathrm{L}, \mathrm{M}$ & NO & $\mathrm{Vc}$ & Vc \\
\hline $\begin{array}{l}\text { 98. Ploceus philippinus (Linnaeus } \\
\text { 1766) }\end{array}$ & Baya Weaver & Babui & $\mathrm{R}$ & Vc & $\mathrm{P}, \mathrm{G}$ & $\mathrm{T}, \mathrm{V}, \mathrm{O}$ & $\mathrm{L}, \mathrm{M}, \mathrm{U}$ & NO & $\mathrm{C}$ & Vc \\
\hline 99. Anthus rufulus (Gmelin 1789) & Paddyfield Pipit & Dhani Tulika & $\mathrm{M}$ & $\mathrm{C}$ & $P, G$ & $\mathrm{O}, \mathrm{Pf}$ & $\mathrm{L}$ & - & $\mathrm{Vc}$ & Vc \\
\hline $\begin{array}{l}\text { 100. Motacilla alba (Linnaeus } \\
\text { 1758) }\end{array}$ & White Wagtail & Sada Khonjan & M & $\mathrm{Fc}$ & $\mathrm{P}, \mathrm{G}$ & $\mathrm{V}, \mathrm{O}$ & $\mathrm{L}$ & - & $\mathrm{C}$ & $\mathrm{Vc}$ \\
\hline $\begin{array}{l}\text { 101. Motacilla flava (Linnaeus } \\
\text { 1758) }\end{array}$ & Yellow Wagtail & $\begin{array}{l}\text { Halud } \\
\text { Khonjan }\end{array}$ & $\mathrm{M}$ & $\mathrm{F}$ & $\mathrm{P}, \mathrm{G}$ & $\mathrm{V}, \mathrm{Bu}$ & $\mathrm{L}$ & - & $\mathrm{Vc}$ & $\mathrm{C}$ \\
\hline
\end{tabular}

Note: IUCN - IUCN (2000), SS - Sarker and Sarker (1988), RK - Khan (1982), R - Resident, M - Migratory, Distribution: G - Gajdhar, P - Pashukhali, Habitat: B - Beel, T - Tree, O - Open field, We - Water edge, V - Village, Bu - Bush, Pf - Paddy field, P - Pond, Re - River edge, Status: Vc - Very Common, C - Common, Fc - Fairly Common, F - Few, r - rare, NO - Not Threatened, DD - Data deficient, VU - Vulnerable, CR - Critically Endangered, O - Occasional, U - Uncertain, Uc - Uncommon, Canopy used: L - Lower Canopy, M - Middle Canopy, U - Upper Canopy 
restricted only to Gajdhar and $6(14.3 \%)$ to Pashukhali (Table 1). The study showed that Gajdhar supported more species, because it got more diversified and undisturbed bird's habitat than Pashukhali.

From the habitat analysis it was found that most of the birds were found to use more than one habitat (Table 2). Out of 101 species, the highest numbers of the species $(78.2 \%)$ were recorded on land, such as; tree $(41 \%)$, village area $(16 \%)$, paddy field $(12 \%)$, open field $(7 \%)$, bush $(2.2 \%)$, etc. Because it was more favourable habitat for feeding, breeding, resting and highly diversified than aquatic and semi-aquatic bodies. The lowest numbers $(5 \%)$ of the birds were recorded from aquatic habitats (Table 2).

A brief analysis of canopy levels showed that most of the species (30.7\%) were recorded in lower canopy. The lowest number of $(3.9 \%)$ species used all three canopy levels. In case of migratory birds, most of the species $(71.4 \%)$ were recorded in lower canopy while most resident $(22.5 \%)$ bird species used both lower and middle canopies. The Gajdhar, Pashukhali were quite rich and diversified habitat for wildlife especially avifauna. During the monsoon the areas go under water and at late monsoon they become muddy with various feeding habitats, which rather invite waders and ducks in large groups.

Acknowledgement: Special thanks to Mr. Tauhid Uddin Ahmed, Former Principal Scientific Officer, IECDR, Mohakhali, Dhaka, Bangladesh for constructive comments and suggestions to enrich this manuscript.

\section{LITERATURE CITED}

AHSAN, M.F. and KHANOM, N. 2005. Birds of the Chittagong University Campus, Chittagong. The Chittagong Univ. J. Sci. 15(1): 77-88.

ALI, S. 1996. The book of Indian birds (12 th revised and centenary edition). Bombay Natural History Society, New Delhi. 345 pp.

ALI, S. and RIPLEY, S.D. 1983. A pictorial guide to the birds of the Indian Subcontinent. Oxford Univ. Press. Delhi. 177 pp. +106 plates.

ALI, S. and RIPLEY, S.D. 1987. Compact handbook of the birds of India and Pakistan. $2^{\text {nd }}$ ed. Oxford University Press, Delhi. 737 pp +104 plates.

GRIMMETT, R., INSKIPP, C. and INSKIPP, T. 1999. Pocket guide to the birds of the Indian Subcontinent. Oxford University Press, New Delhi. 384 pp.

ISLAM, M.T. 1970. Birds of Rangpur (with notes on taxonomy, food and relationships). M.Sc. Thesis (Unpublished). University of Dhaka.

IUCN-BANGLADESH. 2000. Red book of threatened birds of Bangladesh. IUCN-The World Conservation Union, Dhaka. xii+116 pp.

KHAN, M.A.R. 1982. Wildlife of Bangladesh. University of Dhaka, Dhaka. 173 pp.

KHAN, M.M.H. 2005. Species diversity, relative abundance and habitat use of the birds in the Sundarbans East Wildlife Sanctuary, Bangladesh. Forktail. 21: 79-86. 
KHAN, S.I. and NAHER, H. 2009. Birds in Kurigram district of Bangladesh. J. Threatened Taxa. 1(4): 245-250.

SARKER, S.U. and SARKER, N.J. 1988. Wildlife of Bangladesh (a systematic list with status, distribution and habitat). The Richo Printers, Dhaka. 69 pp.

THOMPSON, P.M. and JOHSON, D.L. 2003 Further notable bird records from Bangladesh. Forktail. 19: 85-102.

(Manuscript received on 2 February, 2014; revised on 28 September, 2014) 